\title{
Dietary restraint and weight loss maintenance in members of a commercial weight loss organisation
}

\author{
S. Whybrow ${ }^{1}$, J Westenhoefer ${ }^{2}$, D. Engel ${ }^{2}$, A. McConnon ${ }^{3}$, M. Gibbs ${ }^{1}$, M. Raats ${ }^{3}$ and R. J. Stubbs ${ }^{4}$ \\ ${ }^{1}$ Food, Consumer Behaviour and Health Research Centre, Faculty of Health and Medical Sciences, University of Surrey, \\ Guildford GU2 7XH, ${ }^{2}$ Hamburg University of Applied Sciences, Department of Health Sciences, Germany, \\ ${ }^{3}$ Food, Consumer Behaviour and Health Research Centre, Department of Psychology, University of Surrey, \\ Guildford GU2 $7 X H$ and ${ }^{4}$ Slimming World, Alfreton DE55 4UE
}

This analysis examined the association between restrained eating behaviour and weight loss maintenance in 1428 participants of a slimming organisation who had been members for a mean \pm SD $16 \pm 16$ months. They had lost $13.8 \% \pm 9.2 \%$ of their initial weight and were trying to maintain, or increase, their weight loss during a subsequent 6-month study period.

Data were collected as part of the DiOGenes study ${ }^{(1)}$. Ethical approval was given by the University of Surrey Ethics Committee. Adults were recruited between August 2006 and July 2008 from Slimming World at group meetings and by email. Subjects completed questionnaires at two time points, measurement 1 (M1) at the start of the study and nominally six months later (measurement 2 (M2)). Participants' weights (using calibrated scales) were taken from group records for M1, M2, six months before (measurement 0) and when they initially enrolled. Participants were free to continue following the weight-loss programme as they wished during this study, and there was no intervention other than completing the questionnaires.

Cognitive restraint of eating or dietary restraint, disinhibition and susceptibility to hunger were measured using the Three Factor Eating Questionnaire $^{(2)}$. Dietary restraint is not a single construct, but can be split into flexible and rigid restraint. These two components of restraint were assessed by validated questionnaire ${ }^{(3)}$. Linear regression analysis was used to identify the associations between questionnaire responses and weight change (as a percentage of M1 weight) over the study period.

\begin{tabular}{lccc}
\hline & Correlation & $\mathrm{R}^{2}$ & $p$ \\
\hline Disinhibition of control & +0.085 & 0.7 & 0.020 \\
Susceptibility to hunger & +0.010 & 0.0 & 0.780 \\
Flexible control & -0.046 & 0.2 & 0.207 \\
Rigid control & -0.002 & 0.0 & 0.964 \\
\hline
\end{tabular}

Participants who reported having low levels of disinhibition at the first measurement had more positive weight gains during the following six months. This only explained a small proportion of the variance in weight loss maintenance. Cognitive restraint and its components were not strongly associated with weight loss maintenance.

This work was part of the Diet, Obesity and Genes project (www.diogenes-eu.org) funded by the European Commission (contract \#: Food-CT-2005513946) in the Food Quality and Safety Priority of the Sixth Framework Program.

1. Larsen TM, Dalskov S et al. (2010) "The Diet, Obesity and Genes (Diogenes) Dietary Study in eight European countries - a comprehensive design for long-term intervention." Obesity Reviews 11, 76-91.

2. Stunkard AJ \& Messick S (1985) "The three-factor eating questionnaire to measure dietary restraint, disinhibition and hunger." Journal of Psychosomatic Research 29(1), 71-83.

3. Westenhoefer J, Stunkard AJ, et al. (1999) "Validation of the flexible and rigid control dimensions of dietary restraint." International Journal of Eating Disorders 26(1), 53-64. 